Primeros registros de ocelote en los bosques tropicales de la Sierra Negra de Puebla y la Sierra Mazateca de Oaxaca, México

\title{
First records of ocelot in tropical forests of the Sierra Negra of Puebla and Sierra Mazateca de Oaxaca, Mexico
}

\author{
Rosa Elena Galindo-Aguilar ${ }^{1}$, Laura Angélica Cacelin-Castillo², Octavio César Rosas-Rosas ${ }^{3 *}$, María Guadalupe Bravo- \\ Vinaja ${ }^{1}$, José Luis Alcántara-Carbajal ${ }^{1}$ y Verónica Vázquez-García ${ }^{4}$
}

\author{
1 Ganadería, Recursos Genéticos y Productividad. Colegio de Postgraduados Campus Montecillo. Carretera México-Texcoco Km \\ 36.5, Montecillos 56230. Estado de México, México. E-mail: rosa.galindo@colpos.mx (REGA), gbravo@colpos.mx (MGBV), jlalcant@ \\ colpos.mx (JLAC). \\ ${ }^{2}$ Escuela de Biología, Benemérita Universidad Autónoma de Puebla. 4 sur 104, Puebla, 72000. Puebla, México. E-mail: laucacelin@ \\ gmail.com (LACC). \\ ${ }^{3}$ Colegio de Postgraduados Campus San Luis Potosí, Iturbide 73, Salinas San Luis Potosí, 78600. San Luis Potosí, México. E-mail: \\ octaviocrr@colpos.mx (OCRR). \\ ${ }^{4}$ Desarrollo rural, Socioeconomía, Estadística e Informática. Colegio de Postgraduados Campus Montecillo. Carretera México- \\ Texcoco Km 36.5, Texcoco, 56230. Estado de México, México. E-mail: gveronica@colpos.mx (VVG). \\ ${ }^{*}$ Corresponding author
}

Ocelots are at risk of extinction despite being a wide distribution species. Its presence in Puebla has been recorded, and also the possibility of existence of biological corridor to southeastern Mexico. Part of that corridor is located in the Sierra Negra in Puebla and the Sierra Mazateca in Oaxaca, where there are not previous studies that prove the presence of the ocelot. The Sierra Negra of Puebla and Sierra Mazateca of Oaxaca are contiguous mountain ranges with a mosaic of vegetation that includes tall evergreen forest, cloud forest, rain-fed agriculture and cultivated grasslands. We placed 11-18 camera trap stations spaced every 1-3 km, within a 110 $\mathrm{km}^{2}$ polygon, over three sampling periods. We also applied semistructured interviews to locals to obtain information on wild felids occurrence. Twelve individual ocelots were recorded in the study area, 10 of them through cameras and six through interviews. Ocelots were photographed in fragmented areas near human settlements. The six remaining ocelots registered were four skins, one carcass and one captive animal. Ocelots have been previously documented in disturbed landscapes and near human settlements areas. We believe that there is a resident ocelot population in the study area, thus the Sierra Negra and the Sierra Mazateca are an important component of the proposed biological corridor of the Sierra Madre Oriental.

\begin{abstract}
El ocelote, a pesar de ser una especie de amplia distribución, se encuentra en peligro de extinción. En Puebla, se ha documentado su presencia y la posibilidad de un corredor hacia el sureste mexicano. Parte de dicho corredor se localiza en la Sierra Negra de Puebla y la Sierra Mazateca de Oaxaca, en las cuales no se han realizado estudios que comprueben la presencia del ocelote. La Sierra Negra de Puebla y la Sierra Mazateca de Oaxaca son cadenas montañosas contiguas, las cuales presentan un mosaico de vegetación que incluye selva alta perennifolia, bosque mesófilo de montaña, agricultura de temporal y pastizal inducido. En estas sierras se delimitó un polígono de $110 \mathrm{~km}^{2}$, donde durante tres periodos de muestreo se colocaron de 11 a 18 estaciones de fototrampeo distanciadas entre sí cada 1-3 km. Además se realizaron entrevistas semiestructuradas para documentar la presencia de los felinos silvestres. Se registraron en total 12 individuos de ocelote en la zona de estudio. Por medio de las cámaras se detectaron seis de éstos en zonas fragmentadas y cercanas a asentamientos humanos. A través de entrevistas se documentaron otros seis individuos (cuatro pieles, un cadáver y uno en cautiverio). Se ha documentado con anterioridad la presencia de ocelotes en ambientes perturbados y cercanos a asentamientos humanos. Consideramos que en la zona de estudio existe una población residente de ocelotes; por lo tanto, la Sierra Negra y la Sierra Mazateca son un componente importante del propuesto corredor biológico de la Sierra Madre Oriental.
\end{abstract}

Key words: connectivity, biological corridor, Leopardus pardalis, mammals, carnivore, rainforest, cloud forest.

๑ 2016 Asociación Mexicana de Mastozoología, www.mastozoologiamexicana.org 


\section{Introducción}

El ocelote (Leopardus pardalis) es una especie de amplia distribución en el Neotrópico, donde habita una gran variedad de ecosistemas, entre ellos los bosques tropicales y templados, manglares, matorral xerófilo y matorral desértico micrófilo (Aranda 2005; Martínez-Calderas et al. 2011; Ahumada-Carrillo et al. 2013; Aranda et al. 2014). No obstante, se encuentra en peligro de extinción debido al efecto de las actividades humanas, como la cacería y la destrucción y fragmentación de su hábitat (Caso et al. 2008; SEMARNAT 2010). La Convención sobre el Comercio Internacional de Especies Amenazadas de Fauna y Flora Silvestres (CITES, por sus siglas en inglés) lo incluye en el Apéndice I (CITES 2015) y la Unión Internacional para la Conservación de la Naturaleza y los Recursos Naturales (UICN, por sus siglas en inglés) lo clasifica como LC (least concern) o en "preocupación menor" (IUCN 2015). Su distribución histórica incluye la Sierra Madre Oriental (SMO; Aranda 2005), la cual se encuentra altamente modificada por las actividades antropogénicas (Arriaga et al. 2000). Se ha documentado su presencia en las selvas altas perennifolias del estado de Puebla (Hueytamalco y Plan de Guinea; Ramírez-Bravo et al. 2010) y Oaxaca (San Juan Teponaxtla y los Chimalapas), así como en la selva baja caducifolia (Tehuacán, Puebla; Ramírez-Bravo et al. 2010; Pérez-Irineo y Santos-Moreno 2014), por lo que se ha propuesto la existencia de un corredor biológico a lo largo de la SMO, el cual conecta con el sureste mexicano a través de los estados de Puebla, Veracruz y Oaxaca (Ramírez-Bravo et al. 2010). En dicha zona se ubican la Sierra Negra de Puebla y la Sierra Mazateca de Oaxaca; ambas sierras forman parte de la Región Terrestre Prioritaria 130 (RTP) "Sierras del norte de Oaxaca-Mixe". Esta sierra se caracteriza por mantener selvas altas perennifolias y bosques mesófilos de montaña, así como una alta riqueza de mamíferos y una alta concentración de especies en riesgo (Arriaga et al. 2000; García-Marmolejo et al. 2008). Esta región se considera con potencial para la distribución del ocelote y otros felinos tropicales, incluyendo al jaguar (Lorenzana 2011; Rodríguez-Soto et al. 2011, 2013; Dueñas-López et al. 2015). Sin embargo, no existen registros que corroboren la presencia del ocelote ni la de otros felinos silvestres en esta región en particular.

\section{Materiales y métodos}

El estudio se llevó a cabo en dos municipios: San Sebastián Tlacotepec en la Sierra Negra, Puebla y Santa María Chilchotla en la Sierra Mazateca, Oaxaca. Ambas sierras son dos cadenas montañosas contiguas que presentan un mosaico de vegetación compuesto por agricultura de temporal (16,640 ha), bosque mesófilo de montaña (12,508 ha), selva alta perennifolia (11,943 ha), pastizal cultivado ( 9,758 ha) y bosque de pino (57,44 ha; CONABIO 2012). El fragmento más grande de vegetación natural consta de 3,200 ha de bosque mesófilo de montaña, el cual se localiza en la Sierra Negra. Realizamos entrevistas informales y semiestructuradas en 23 comunidades de la zona de estudio entre octubre de 2013 y octubre de 2014, para conocer el estado de conservación del jaguar y sus presas en los bosques tropicales de la zona. Se utilizó la técnica conocida como bola de nieve, la cual consiste en localizar a través de las entrevistas, a los pobladores que tengan conocimientos profundos sobre el tema de interés (Sandoval 2002); en este caso, información sobre felinos silvestres o posesión de sus derivados. Como parte de un estudio para determinar presencia y abundancia del jaguar y sus presas se colocaron entre 11 y 18 estaciones de fototrampeo en selva alta perennifolia, bosque mesófilo de montaña y acahuales. El diseño de muestreo se realizó siguiendo la metodología de Medellín et al. (2006), Díaz-Pulido y Payán (2012) y Noss et al. (2013) para el jaguar; esto es, un área mayor a $100 \mathrm{~km}^{2}$ y una distancia entre las estaciones de $3 \mathrm{~km}$. El muestreo se realizó durante tres meses, de julio a octubre de 2014 con 18 estaciones: cuatro en la Sierra Negra y ocho en la Sierra Mazateca. Seis estaciones fueron dobles, éstas se colocaron en donde los cazadores refirieron avistamientos recientes de rastros del jaguar 
o sus presas potenciales, y se comprobó el rastro (los rastros comprobados siempre fueron de las presas potenciales del jaguar: Pecari tajacu, Mazama temama), dos se colocaron en la Sierra Negra y cuatro en la Sierra Mazateca. En las estaciones dobles se colocó perfume Obssesion ${ }^{\circledR}$ como atrayente para el jaguar. Como parte de un estudio piloto, se realizaron dos muestreos previos de un mes, uno en diciembre de 2013, en el que se operaron 18 estaciones en la Sierra Negra (1 km entre estaciones) y el otro en abril de 2014 con 11 estaciones (cuatro en la Sierra Negra y siete en la Sierra Mazateca espaciadas cada $3 \mathrm{~km}$ ). Los datos obtenidos de dichos muestreos también forman parte de los resultados presentados en este estudio. La ubicación de las cámaras en cada una de las temporadas de muestreo cambio dependiendo de las condiciones en campo, por lo tanto, al final se lograron algunos registros en puntos con distancias mínimas de $200 \mathrm{~m}$ en línea recta. Las cámaras utilizadas fueron: StealthCam ${ }^{\circledast}$ Delta8 modelo STC-Q8X/STC-D8BZ, Texas, EUA; Cuddeback $^{\circledR}$ Ambush Black Flash Model 1194, Wisconsin, EUA; Bushnell ${ }^{\circledast}$ Trophy Cam HD Essential 119736C, Kansas, EUA y Covert ${ }^{\circledR}$, Kentucky, EUA. Para determinar el esfuerzo total de muestreo se multiplicó el número de cámaras trampa activas por los días de muestreo en cada una de las temporadas; lográndose un esfuerzo total de 1,745 días trampa. No logramos obtener fotografías del jaguar, pero sí de ocelote, y dada la importancia de su registro en la zona, se decidió examinar las fotografías. Para propósitos del análisis se utilizaron solo aquellas fotografías consideradas como eventos independientes, de acuerdo a los criterios establecidos por O'Brien et al. (2003). Para identificar individuos se utilizó el patrón de manchas; pero dado que se obtuvieron fotografías de ambos flancos de un individuo, se decidió utilizar únicamente las fotografías del flanco derecho.

\section{Resultados}

Obtuvimos un total de 31 fotografías independientes de ocelote en 13 estaciones: 10 en la Sierra Negra y tres en la Sierra Mazateca, la mayoría con una separación mayor a $1.5 \mathrm{~km}$ en línea recta (Figura 1). Se lograron identificar seis individuos en cinco de las 13 estaciones (dos en una misma estación), cinco individuos de sexo indeterminado y una cría. La hembra de la cría no pudo ser diferenciada por el patrón de manchas, debido a la baja calidad de la foto, por lo que no se incluyó en los resultados. Se nombraron LP (Leopardus pardalis) y se les asignó un número consecutivo (Tabla 1; Figura 2). En ocho estaciones, por la calidad de la fotografía o por el flanco en el que fue tomada (izquierdo), no fue posible determinar si se trataba de individuos distintos a los ya identificados. Mediante las entrevistas semiestructuradas obtuvimos seis registros adicionales. En la Sierra Mazateca nos mostraron tres pieles en tres comunidades: Dolores, Agua de Paxtle y Rio Sapo; un cadáver congelado para taxidermia en Rio Sapo y un individuo en cautiverio en San Miguel Nuevo. En la Sierra Negra nos mostraron una piel en Mazatzongo de Guerrero. Por consiguiente, registramos un total de 12 ocelotes en el área de estudio, cinco en la Sierra Negra, Puebla y siete en la Sierra Mazateca, Oaxaca (Tabla 1), éstos ocurrieron en zonas fragmentadas, con presencia de selva alta perennifolia, bosque mesófilo de montaña y acahuales y cercanos a los asentamientos humanos, entre 1 y 4 km en línea recta (Figura 1).

\section{Discusión y conclusiones}

Los resultados de la presente investigación coinciden con los de Michalski et al. (2010), quienes indican que el ocelote tiene tolerancia a ambientes fragmentados. Registramos 12 ocelotes (seis en las estaciones de fototrampeo y seis por evidencia física) en una zona fragmentada, lo cual destaca al compararlos con los nueve ocelotes documentados en la segunda extensión más grande de bosque tropical en México, los Chimalapas (Arriaga et al. 2000; Pérez-Irineo y Santos-Moreno 2014). Debido a que no fue posible diferenciar a los individuos que se presentaron en todas las estaciones, creemos que el número de ocelotes podría estar subestimado. Consideramos que la 


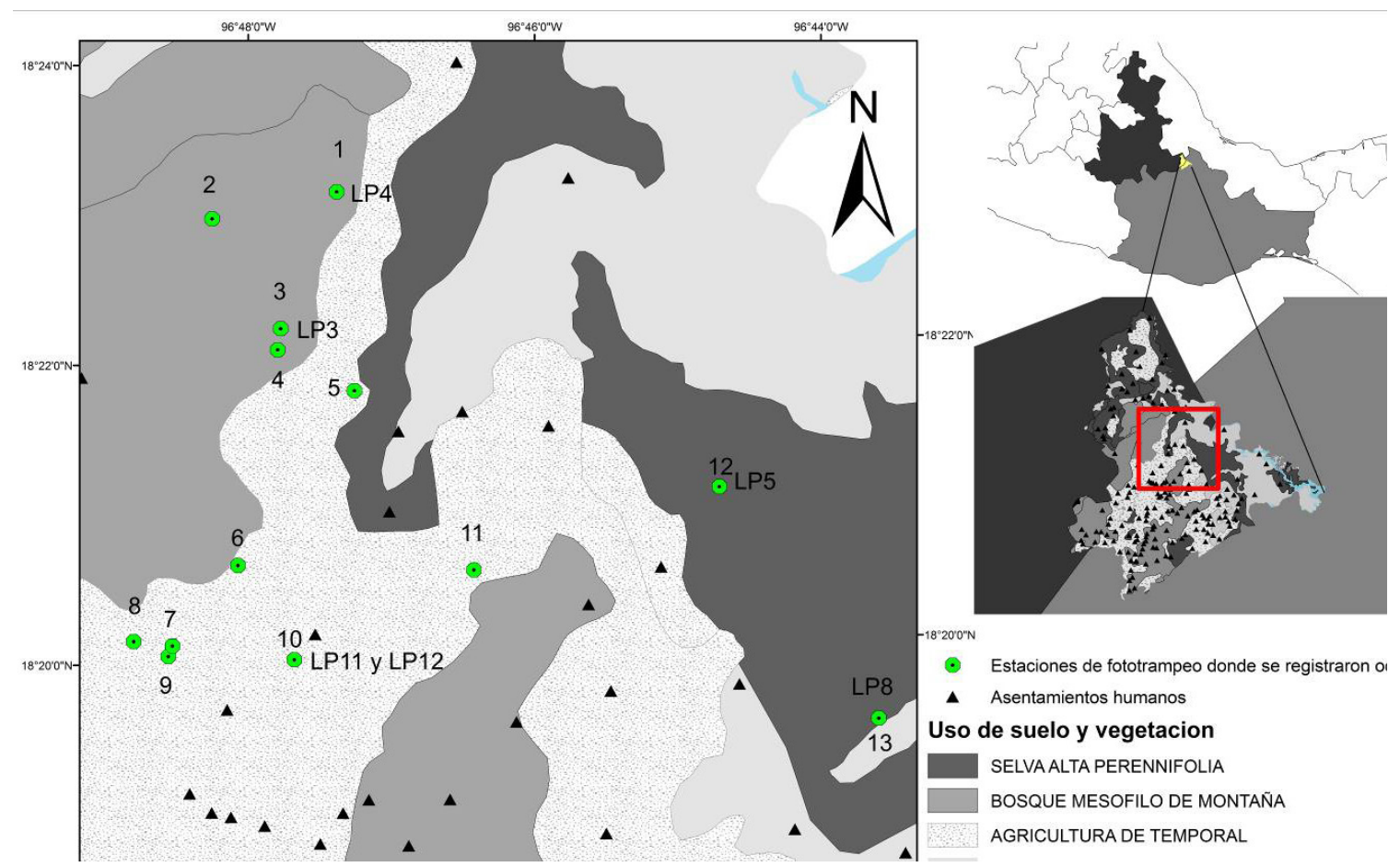

Figura 1. Estaciones de fototrampeo donde se registraron ocelotes (Leopardus pardalis) y posición de los individuos identificados por cámaras trampa en los bosques tropicales de la Sierra Negra de Puebla y Sierra Mazateca de Oaxaca, México. LP = ocelotes registrados, ver Tabla 1 para información de los mismos.

Tabla 1. Localización de registros de ocelote (Leopardus pardalis) en la Sierra Negra de Puebla y la Sierra Mazateca de Oaxaca, México. Undividuo (Ind), Altitud sobre el nivel del mar (Alt). Tipo de vegetacion (Veg)

\begin{tabular}{|c|c|c|c|c|c|c|c|}
\hline \multirow[b]{2}{*}{ Ind } & \multirow{2}{*}{ Localidad/Municipio/Estado } & \multicolumn{2}{|c|}{ Ubicación Geográfica } & \multirow[b]{2}{*}{ Alt } & \multirow{2}{*}{ Veg } & \multirow{2}{*}{$\begin{array}{l}\text { Fecha } \\
\text { Hora }\end{array}$} & \multirow{2}{*}{ Tipo de registro } \\
\hline & & $\begin{array}{l}\text { Latitud } \\
\text { UTM }\end{array}$ & $\begin{array}{l}\text { Longitud } \\
\text { UTM }\end{array}$ & & & & \\
\hline LP1 & $\begin{array}{l}\text { Mazatzongo de Guerrero, San } \\
\text { Sebastián Tlacotepec, Puebla }\end{array}$ & 18.2939 & -96.7769 & 359 & acahual & $29 / 10 / 2013$ & piel \\
\hline LP2 & $\begin{array}{l}\text { Dolores, Santa María } \\
\text { Chilchotla, Oaxaca }\end{array}$ & 18.3586 & -96.7978 & 198 & $\begin{array}{l}\text { selva alta } \\
\text { perennifolia }\end{array}$ & $02 / 12 / 2013$ & piel \\
\hline LP3 & $\begin{array}{l}\text { Ojo de Agua, San Sebastián } \\
\text { Tlacotepec, Puebla }\end{array}$ & 18.3676 & -96.7903 & 1,117 & $\begin{array}{l}\text { selva alta } \\
\text { perennifolia }\end{array}$ & $\begin{array}{l}20 / 12 / 2013 \\
23: 45\end{array}$ & cámara trampa \\
\hline LP4 & $\begin{array}{l}\text { Vista Hermosa, San Sebastián } \\
\text { Tlacotepec, Puebla }\end{array}$ & 18.3849 & -96.7470 & 1,114 & $\begin{array}{l}\text { selva alta } \\
\text { perennifolia }\end{array}$ & $\begin{array}{c}10 / 04 / 2014 \\
11: 29\end{array}$ & cámara trampa \\
\hline LP5 & $\begin{array}{l}\text { Santa Eustolia, Santa María } \\
\text { Chilchotla, Oaxaca }\end{array}$ & 18.3506 & -96.7140 & 221 & acahual & $\begin{array}{c}14 / 07 / 2014 \\
14: 20\end{array}$ & cámara trampa \\
\hline LP6 & $\begin{array}{l}\text { Agua de Paxtle, Santa María } \\
\text { Chilchotla, Oaxaca }\end{array}$ & 18.3026 & -96.7892 & 291 & $\begin{array}{l}\text { selva alta } \\
\text { perennifolia }\end{array}$ & 05/07/2014 & piel \\
\hline LP7 & $\begin{array}{l}\text { San Miguel Nuevo, Santa } \\
\text { María Chilchotla, Oaxaca }\end{array}$ & 18.3183 & -96.7294 & 1,181 & $\begin{array}{l}\text { bosque } \\
\text { mesófilo de } \\
\text { montaña }\end{array}$ & 03/08/2014 & vivo \\
\hline LP8 & $\begin{array}{l}\text { Barranca Seca, Santa María } \\
\text { Chilchotla, Oaxaca }\end{array}$ & 18.3242 & -96.7164 & 119 & acahual & $\begin{array}{l}\text { 07/08/2014 } \\
05: 15\end{array}$ & cámara trampa \\
\hline LP9 & $\begin{array}{l}\text { Rio Sapo, Santa María } \\
\text { Chilchotla, Oaxaca }\end{array}$ & 18.2743 & -96.7164 & 400 & $\begin{array}{l}\text { bosque } \\
\text { mesófilo de } \\
\text { montaña }\end{array}$ & 04/08/2014 & piel \\
\hline LP10 & $\begin{array}{l}\text { Rio Sapo, Santa María } \\
\text { Chilchotla, Oaxaca }\end{array}$ & 18.2743 & -96.7972 & 400 & acahual & 04/08/2014 & cadáver congelado \\
\hline LP11 & $\begin{array}{l}\text { Cruztitla, San Sebastián } \\
\text { Tlacotepec, Puebla }\end{array}$ & 18.3330 & -96.7972 & 928 & $\begin{array}{l}\text { selva alta } \\
\text { perennifolia }\end{array}$ & $\begin{array}{l}17 / 08 / 2014 \\
05: 54\end{array}$ & cámara trampa \\
\hline LP12 & $\begin{array}{l}\text { Cruztitla, San Sebastián } \\
\text { Tlacotepec, Puebla }\end{array}$ & 18.3330 & -96.7972 & 928 & $\begin{array}{l}\text { selva alta } \\
\text { perennifolia }\end{array}$ & $\begin{array}{c}02 / 10 / 2014 \\
19: 58\end{array}$ & cámara trampa \\
\hline
\end{tabular}




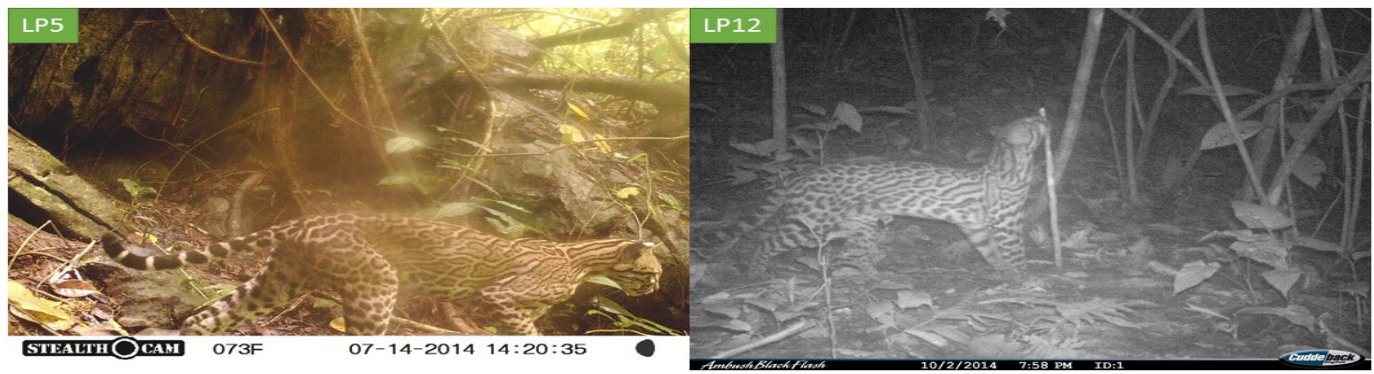

Figura 2. Ocelotes (Leopardus pardalis) diferenciados por su patrón de manchas en la Sierra Negra de Puebla y la Sierra Mazateca de Oaxaca, México.

probabilidad de que se fotografíe el mismo individuo en estaciones espaciadas por más de $2 \mathrm{~km}$ es baja; dado que en un arreglo de estaciones separadas por $1.5 \mathrm{~km}$ solo tres individuos de 15 fueron fotografiados en más de una estación (Martínez-Hernández et al. 2014) y que el ámbito hogareño del ocelote es de 1.8 a $4.6 \mathrm{~km}^{2}$, el cual se traslapa con el de otros individuos (Torres-Romero 2009). Por lo tanto, cabe la posibilidad de considerar, por lo menos, tres individuos más, los de las estaciones 2,8 y 11, las cuales tuvieron una separación de $2 \mathrm{~km}$ o más en línea recta de aquellas estaciones donde se logró la identificación plena de individuos (Figura 1). De esta manera obtendríamos un total de 15 ocelotes. Los registros de los ocelotes en los Chimalapas ocurrieron a distancias de entre 3 y $5 \mathrm{~km}$ de asentamientos humanos (Pérez-Irineo y Santos-Moreno 2014); distancias similares a las de este estudio: 1 a 4 km. La proximidad del ocelote a los poblados puede obedecer a la presencia de presas como el serete (Dasyrpocta mexicana), el cual tiene tolerancia a la presencia humana (Espinosa-Andrade 2012) y presenta el mayor índice de abundancia en la zona de estudio (Galindo-Aguilar 2015), o el tejón (Nasua narica), que se encuentra comúnmente en las milpas (Galindo-Aguilar 2015). El número de registros, la presencia de una hembra con cría y un cachorro en cautiverio sugiere la existencia de una población residente de ocelotes en los bosques tropicales de la Sierra Negra y la Sierra Mazateca y robustece la existencia de conectividad entre las poblaciones de ocelote al sur de la SMO y el sureste mexicano (y potencialmente para otras especies de felinos) mediante el propuesto corredor biológico de la Sierra Madre Oriental (Dueñas-López et al. 2015).

\section{Agradecimientos}

Agradecemos al Consejo Nacional de Ciencia y Tecnología por la beca de maestría otorgada a la primera autora. Al Programa de Conservación de Especies en Riesgo de la CONANP por el apoyo financiero. Al Colegio de Posgraduados por el financiamiento a través del fideicomiso no. 167304; así como a todos los involucrados en el monitoreo de campo, especialmente a los pobladores quienes aportaron información y guía a través del bosque tropical.

\section{Literatura citada}

Ahumada-Carrillo, I. T., J. C. Arenas-Monroy, y M. A. Íñiguez. 2013. Presence of the ocelot (Leopardus pardalis) in northern Jalisco, Mexico. Revista Mexicana de Biodiversidad 84:718-721.

Aranda, M. 2005. Ocelote. Pp. 359-361 en Los mamíferos silvestres de México (Ceballos, G., y G. Oliva, eds.). CONABIO, Fondo de Cultura Económica. Ciudad de México, México. 
Aranda, M., F. Botello, E. Martínez-Meyer, y A. Pineda. 2014. Primer registro de ocelote (Leopardus pardalis) en el Parque Nacional Lagunas de Zempoala, Estado de México y Morelos, México. Revista Mexicana de Biodiversidad 85:1300-1302.

Arriaga, L., J. Espinoza, C. Aguilar, E. Martínez, L. Gómez, y E. Loa (coords.). 2000. Regiones terrestres prioritarias de México. Comisión Nacional para el Conocimiento y Uso de la Biodiversidad. Ciudad de México, México.

Caso, A., C. López-González., E. Payan, E. Eizirik, T. de Oliveira, R. Leite-Pitman, M. Kelly, y C. Valderrama. 2008. Leopardus pardalis. The IUCN Red List of Threatened Species. Version 2015.1. www.iucnredlist.org. Consultado: 15 de julio de 2015.

Cites (Convención sobre el Comercio Internacional de Especies Amenazadas de Fauna y Flora Silvestres). 2015. Apéndices I, II y III. www.cites.org/eng/app/appendices.php. Consultado: 15 de julio de 2015.

CONABio (Comisión Nacional para el Conocimiento y Uso de la Biodiversidad). 2012. Portal de Geoinformación. Sistema Nacional de Información sobre Biosdiversidad. http://www. conabio.gob.mx/informacion/gis/

Díaz-Pulido, A., y E. Payán. 2012. Manual de fototrampeo: una herramienta de investigación para la conservación de la biodiversidad en Colombia. Instituto de Investigaciones de Recursos Biológicos Alexander von Humboldt y Panthera Colombia. Bogotá, Colombia.

Dueñas-López, G., O. C. Rosas-Rosas, L. Chapa-Vargas, L. Bender, L. A. Tarango-Arámbula, J. F. Martínez-Montoya, y J. L. Alcántara-Carbajal. 2015. Connectivity among jaguar populations in the Sierra Madre Oriental, México. Therya 7:449-468.

Espinosa-AndRADE, S. 2012. Road development, bushmeat extraction and jaguar conservation in Yasuni Biosphere Reserve-Ecuador. Dissertation Doctor of Phylosophy. University of Florida. Gainesville, EE. UU.

Galindo-Aguilar, R. E. 2015. Distribución, abundancia y conservación del jaguar y sus presas en los bosques tropicales de dos municipios de la Sierra Negra de Puebla y la Sierra Mazateca de Oaxaca, México. Tesis de Maestría. Colegio de Postgraduados. Montecillos, Estado de México.

García-Marmolejo, G., T. Escalante, y J. Morrone. 2008. Establecimiento de prioridades para la conservación de mamíferos terrestres neotropicales de México. Mastozoología Neotropical 15:41-65.

IUCN (International Union for Conservation of Nature). 2015. Red List of threatened species. IUCN, Gland.www.iucnredlist.org. Consultado: 15 de julio de 2015.

Lorenzana, G. 2011. Modelado de la distribución y abundancia del jaguar (Panthera onca) en la Reserva de la Biosfera Sierra Gorda Querétaro. Tesis de Maestría. Xalapa, Veracruz.

Martínez-Calderas, J., O. C. Rosas-Rosas, J. Martínez-Montoya, L. Tarango-Arámbula, F. Clemente-Sánchez, M. Crosby-Galván, y M. Sánchez-Hermosillo. 2011. Distribución del ocelote (Leopardus pardalis) en San Luis Potosí, México. Revista Mexicana de Biodiversidad 82:997-1004.

Martínez-Hernández, A., O. C. Rosas-Rosas, F. Clemente-Sánchez, L. Tarango-Arámbula, J. Palacio-Núñez, L. Bender, y J. Herrera-Haro. 2014. Density of threatened ocelot Leopardus pardalis in the Sierra Abra-Tanchipa Biosphere Reserve, San Luis Potosí, México. Oryx 49:619-625.

Medellín, R., D. Azuara, L. Maffei, H. Zarza, H. Bárcenas, E. Cruz, R. Legaria, I. Lira-Torres, G. Ramos, O. Gaona, y S. Ávila. 2006. Censos y monitoreo. Pp. 25-35 en Memorias del Primer Simposio El Jaguar Mexicano en el Siglo XXI: Situación Actual y Manejo (Chávez, C., y G. Ceballos, eds.). CONABIO-Alianza WWF/TELCEL- Universidad Nacional Autónoma de México. Ciudad de México, México. 
Michalski, F., D. NorRis, y J. Metzger. 2010. Do ocelots use riparian corridors to move across a fragmented landscape? Catnews 53:4-7.

Noss, A., J. Polisar, L. Maffei, R. García, y S. Silver. 2013. Evaluando la densidad de jaguares con trampas cámara. Programa para la Conservación del Jaguar Programa para Latinoamérica y el Caribe Wildlife Conservation Society. New York, EE.UU.

O'Brien, T., M. Kinnaird, y H. WiBisono. 2003. Crouching tigers, hidden prey: Sumatran tiger and prey populations in a tropical forest landscape. Animal Conservation 6:131-139.

Pérez-Irineo, G., y A. Santos-Moreno. 2014. Density, distribution, and activity of the ocelot Leopardus pardalis (Carnivora: Felidae) in Southeast Mexican rainforests. Revista de Biología Tropical 62:1421-1432.

Ramírez-Bravo, E., E. Bravo-Carrete, C. Hernandez-Santín, S. Schinkel, y K. Chris. 2010. Ocelot (Leopardus pardalis) distribution in the state of Puebla, Central Mexico. Therya 1:111120.

Rodríguez-Soto, C., O. Monroy-Vilchis, L. Maiorano, L. Boitani, J. C. Faller, M. Briones, R. Nuñéz, O. Rosas-Rosas, G. Ceballos, y A. Falcuccl. 2011. Predicting potential distribution of the jaguar (Panthera onca) in México: identification of priority areas for conservation. Diversity and Distributions 17:350-361.

Rodríguez-Soto, C., O. Monroy-Vilchis, y M. Zarco-Gonzales. 2013. Corridors for jaguar (Panthera onca) in Mexico: Conservation strategies. Journal for Nature Conservation 21:438-443.

Sandoval, A. 2002. Investigación cualitativa. Instituto Colombiano para el fomento de la educación superior ICFES. Bogotá, Colombia.

SEMARNAT (Secretaría de Medio Ambiente y Recursos Naturales). 2010. Norma Oficial Mexicana NOM-059-ECOL-2010. Protección ambiental, especies de flora y fauna silvestres de México, categorías de riesgo y especificaciones para su inclusión, exclusión o cambio, y lista de especies en riesgo.

Torres-Romero, E. 2009. Densidad, abundancia, uso de hábitat y patrones de actividad del ocelote (Leopardus pardalis) en la zona noreste del Estado de Quintana Roo: Estudio usando cámaras trampa. Tesis de Maestría. El Colegio de la Frontera Sur. San Cristóbal de Las Casas, Chiapas.

Submited: August 10, 2015

Reviewed: September 10, 2015

Accepted: Decembre 6, 2015

Associated editor: Consuelo Lorenzo 
PRIMER REGISTRO DE OCELOTE EN LAS SIERRAS DEL NORTE DE OAXACA-MIXE 